\title{
BMJ Open Association between vomiting in the first trimester and preterm birth: a retrospective birth cohort study in Wuhan, China
}

\author{
Ronghua Hu, ${ }^{1}$ Yawen Chen, ${ }^{1}$ Yiming Zhang, ${ }^{1}$ Zhengmin Qian, ${ }^{2}$ Yan Liu, ${ }^{1}$ \\ Michael G Vaughn, ${ }^{2}$ Shunqing Xu, ${ }^{3}$ Tongzhang Zheng, ${ }^{4}$ Mingzhu Liu, ${ }^{1}$ Bin Zhang ${ }^{1}$
}

To cite: Hu R, Chen $Y$, Zhang Y, et al. Association between vomiting in the first trimester and preterm birth: a retrospective birth cohort study in Wuhan, China. BMJ Open 2017;7:e017309. doi:10.1136/ bmjopen-2017-017309

- Prepublication history and additional material for this paper are available online. To view, please visit the journal (http:// dx.doi.org/10.1136/bmjopen2017-017309).

$\mathrm{RH}$ and $\mathrm{YC}$ contributed equally.

Received 14 April 2017

Revised 12 August 2017

Accepted 17 August 2017

\section{CrossMark}

${ }^{1}$ Health Surveillance

Department, Wuhan Children's Hospital (Wuhan Maternal and Child Healthcare Hospital), Tongji Medical College, Huazhong University of Science and Technology, Wuhan, China ${ }^{2}$ Department of Epidemiology, College for Public Health and

Social Justice, Saint Louis University, Saint Louis, Missouri, USA

${ }^{3}$ Key Laboratory of Environment and Health Department, School of Public Health, Tongji Medical College, Huazhong University of Science and Technology, Wuhan, China

${ }^{4}$ Department of Epidemiology, School of Public Health, Brown University, Providence, Rhode Island, USA

Correspondence to

Dr Bin Zhang;

mchwhzb@163.com

\section{ABSTRACT}

Objective Although vomiting in the first trimester has been reported to be associated with preterm birth (PTB), findings supporting this association remain inconsistent. Our aim was to assess the association between vomiting and PTB, as well as evaluate if the association is modified by pre-pregnancy body mass index (BMI).

Design A retrospective cohort study.

Setting Wuhan, a central city of China.

Participants A total of 317463 pregnant women who had a live, singleton newborn from 1 January 2010 to 23 May 2016 were enrolled in our study.

Main outcome measure PTB was defined as gestational age $<37$ gestational weeks. Gestational age was calculated using reports from mothers based on the first day of their last menstrual period. An ultrasound was routinely used to determine gestational age before 12 gestational weeks.

Results Of the 317463 pregnant women, $29.88 \%$ (94 857) experienced vomiting in the first trimester and $5.00 \%$ (15 889) experienced a PTB. Vomiting in the first trimester increased the risk for PTB and the multivariable adjusted OR was 1.05 (95\% Cl 1.02 to 1.09). In the stratified analyses, the association of vomiting in the first trimester was significant among underweight women (adjusted $\mathrm{OR}=1.08,95 \% \mathrm{Cl} 1.04$ to 1.17) and normal pre-pregnancy $\mathrm{BMl}$ women (adjusted $\mathrm{OR}=1.06,95 \% \mathrm{Cl} 1.02$ to 1.11 ), but not in overweight women (adjusted $\mathrm{OR}=1.01,95 \% \mathrm{Cl} 0.90$ to 1.14 ) and obese women (adjusted $\mathrm{OR}=0.93,95 \% \mathrm{Cl}$ 0.73 to 1.19$)$.

Conclusions Our study indicates that vomiting in the first trimester was associated with PTB. Additionally, women with underweight and normal pre-pregnancy BMI who experienced vomiting are more likely to have a PTB.

\section{INTRODUCTION}

Nausea and vomiting in pregnancy (NVP) is a collection of symptoms composed of nausea alone or nausea in combination with vomiting. ${ }^{1}$ This condition typically starts prior to 9 weeks of gestation and subsides by the end of the first trimester; however, this condition has been shown to continue into the second trimester in up to $25 \%$ of pregnant women

\section{Strengths and limitations of this study}

- The present study is a cohort study, which enrolled a large population of 317463 pregnant women.

- This is the first study exploring the relationship between vomiting in the first trimester and preterm birth (PTB) in Chinese women by pre-pregnancy body mass index (BMI) status.

- Vomiting symptoms were based on selfreported data, which may be a possible source of bias.

- The prevalence of overweight and obesity was relatively low in our sample, and the relationship between vomiting and PTB in women with a high pre-pregnancy BMI needs to be further assessed.

and for $0.3 \%-2.3 \%$ into the third trimester. ${ }^{2}$ NVP has an important effect on both individuals and society as it causes emotional distress, depression and can have a negative effect on a woman's activities and relationships. ${ }^{34} \mathrm{NVP}$ also has been found to lead to loss of time at work and a decrease in job productivity. ${ }^{5}$ The aetiology of NVP remains unknown, although it is currently believed to be related to early pregnancy hormones. ${ }^{6}$

Almost $70 \%$ of women worldwide experience NVP. ${ }^{7}$ The average rate of vomiting only in early pregnancy is $47.1 \%$, and the reported rates generally range from $22.3 \%$ to $63.5 \%{ }^{8}$ A Norwegian cohort investigation reported that the rate of NVP was $33 \%$ among 51675 women. ${ }^{9}$ Källén $e t$ al studied the occurrence of NVP via questionnaires in 3675 Swedish pregnant women and observed that $38.3 \%$ of pregnant women reported vomiting in early pregnancy. ${ }^{10}$ One previous meta-analysis, which included 23 studies comprising 67602 women, ${ }^{11}$ estimated the rate of NVP in the USA to be as high as $68.6 \%$. Meanwhile, studies on the rate of NVP in China are few and are limited by their small sample sizes. 


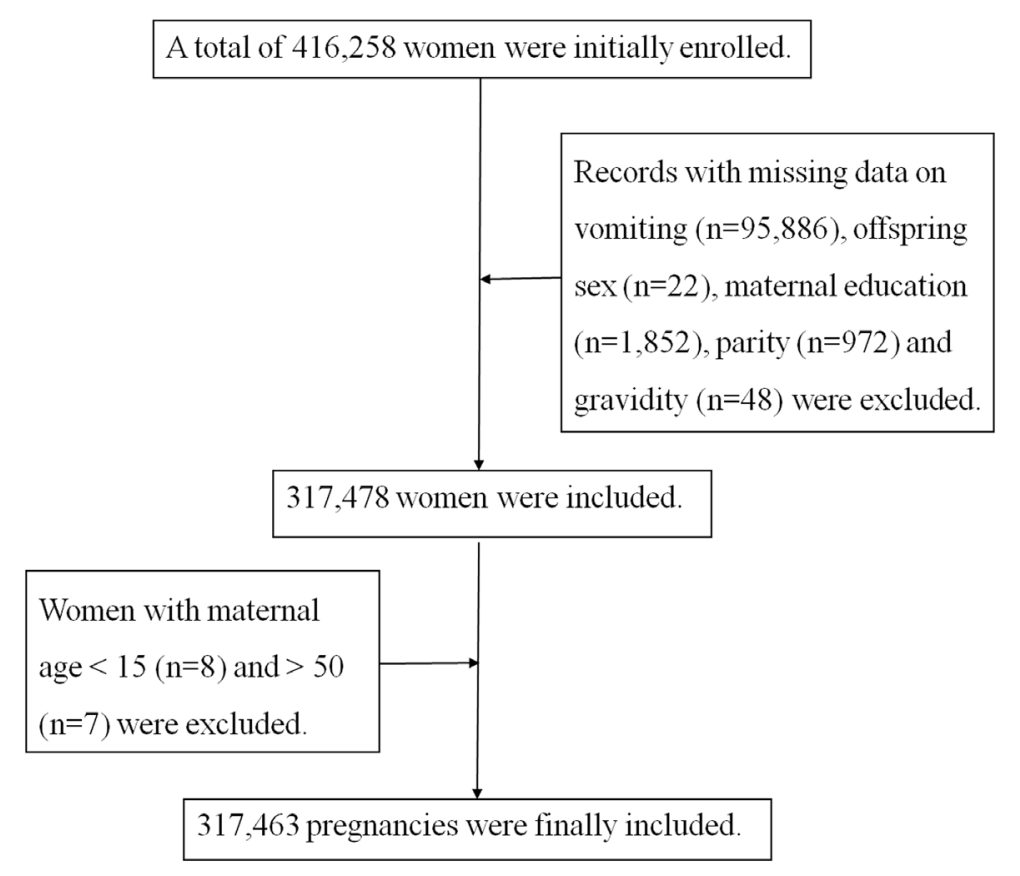

Figure 1 Flow chart of population selection.

Chin ${ }^{12}$ conducted a study in a district hospital in Hong Kong in 1989 and found that the incidence of NVP was $74.9 \%$ among 1453 patients. Another cross-sectional study conducted in a university obstetric unit in Hong Kong revealed that the prevalence of NVP was $90.9 \%$ among 396 women. ${ }^{13}$

In previous studies, NVP was not found to be associated with primary maternal diseases such as gastrointestinal infections or allergies. ${ }^{8}$ Recently, there was a resurgence of interest in topics related to NVP, such as death, ${ }^{14}$ pregnancy complications ${ }^{15}$ and birth outcomes. ${ }^{16}$ Some studies reported that women with NVP were more likely to have a preterm birth (PTB) compared with symptom-free women. ${ }^{15} 1718$ Still, other studies have found no association between this phenomenon. ${ }^{6}{ }^{19}$ However, Czeizel and Puhó have suggested that women with NVP had a lower risk of PTB. ${ }^{20}$ A Norway prospective cohort study involving 51675 pregnant women found nausea to decrease the risk for PTB by $14 \%$ but did not find NVP associated with PTB. ${ }^{9}$

To our knowledge, there has been only one casecontrol study, which was conducted in Chinese pregnant women, that addressed the relationship between PTB and a severe form of NVP called hyperemesis gravidarum. ${ }^{19}$ Information about the association between vomiting in the first trimester and risk of PTB in China is unclear. Furthermore, there has not been a study in which the population was grouped according to pre-pregnancy body mass index (BMI) to determine its contribution to the identification of the association between NVP and risk of PTB. Thus, our objective in the present study is to explore the association between vomiting in the first trimester and PTB as well as assess whether pre-pregnancy BMI modifies this association using a birth cohort study in Wuhan, China.

\section{MATERIALS AND METHODS Study population}

A retrospective cohort study was conducted in Wuhan China, using data from the Wuhan Maternal and Child Health Management Information System (WMCHMIS). The WMCHMIS was introduced in our previous study. ${ }^{21}$ Enrolled women included every pregnant woman who had a live, singleton newborn from 1 January 2010 to 23 May 2016. The study has been approved by the Wuhan Children's Hospital (Wuhan Maternal and Child Healthcare Hospital).

A total of 416258 women were initially enrolled. We then excluded records with missing data on vomiting ( $\mathrm{n}=95886)$, offspring sex $(\mathrm{n}=22)$, maternal education $(\mathrm{n}=1852)$, parity $(\mathrm{n}=972)$ and gravidity $(\mathrm{n}=48)$. Moreover, we also excluded those with a maternal age less than 15 $(n=8)$ and more than $50(n=7)$. The final study population consisted of 317463 pregnancies or $76.27 \%$ of the initial population.

\section{Variables}

Vomiting in the first trimester was assessed when pregnant women first visited the women and children healthcare centres. Maternal age, education, parity, gravidity, pre-pregnancy weight and height were self-reported at their first antenatal care visit, whereas offspring sex and other obstetric information were provided by midwives 


\begin{tabular}{|c|c|c|c|c|c|}
\hline & \multicolumn{2}{|c|}{ Preterm $(n=15889,5.00 \%)$} & \multicolumn{2}{|c|}{$\begin{array}{l}\text { Full-term } \\
(\mathrm{n}=301574,95.00 \%)\end{array}$} & \multirow[b]{2}{*}{ p Value } \\
\hline & $\mathbf{n}$ & $\%$ & $\mathbf{n}$ & $\%$ & \\
\hline$<25$ & 3091 & 3.62 & 82224 & 96.38 & $<0.001$ \\
\hline $25-34$ & 11055 & 5.17 & 202692 & 94.83 & \\
\hline Less than high school & 3519 & 4.55 & 73737 & 95.45 & $<0.001$ \\
\hline High school & 5010 & 4.71 & 101458 & 95.29 & \\
\hline College & 7360 & 5.50 & 126379 & 94.50 & \\
\hline \multicolumn{6}{|l|}{ Offspring sex } \\
\hline Nullipara & 11948 & 4.65 & 245121 & 95.35 & $<0.001$ \\
\hline Multipara & 3941 & 6.53 & 56453 & 93.47 & \\
\hline \multicolumn{6}{|l|}{ Gravidity } \\
\hline$<3$ & 11471 & 4.59 & 238259 & 95.41 & $<0.001$ \\
\hline$\geq 3$ & 4418 & 6.52 & 63315 & 93.48 & \\
\hline \multicolumn{6}{|c|}{ Pre-pregnancy BMI $\left(\mathrm{kg} / \mathrm{m}^{2}\right)$} \\
\hline Underweight $(<18.5)$ & 3118 & 4.21 & 70916 & 95.79 & $<0.001$ \\
\hline Normal (18.5-23.9) & 11083 & 5.03 & 209246 & 94.97 & \\
\hline
\end{tabular}

BMI, body mass index.

documented in WMCHMIS. Information was audited by clinicians and obstetric nurses, and a warning would be activated when illogical data were input into WMCHMIS.

Vomiting was dichotomised into yes and no response formats. Maternal age was categorised into three groups: younger than 25, 25-34 years and 35 years and older. A proxy for socioeconomic status was the maternal education which was categorised into three groups: less than high school, high school and college. By parity, women were dichotomised into nullipara and multipara. Regarding gravidity, it was divided into less than three times and three times and more. Pre-pregnancy BMI was calculated as weight/ height $^{2}$ and grouped into four categories according to Chinese standard of weight for adults. $^{22}$

The main outcome variable was PTB. PTB was defined as gestational age $<37$ gestational weeks. ${ }^{23}$ Gestational age was calculated using reports from mothers based on the first day of their last menstrual period. An ultrasound was routinely used to determine gestational age before 12 gestational weeks.

\section{Statistical analysis}

Results were presented as frequencies (\%). Difference between PTB group and full-term group was assessed by $\mathrm{X}^{2}$ tests. A logistic regression model was used to estimate the association between vomiting in the first trimester and PTB. Results were adjusted for confounders including maternal age, education, parity, gravidity, pre-pregnancy BMI and offspring sex in accordance with previous studies. ${ }^{92}$ We also employed a stratified analysis by pre-pregnancy BMI, and the confounders adjusted in stratified analysis were maternal age, education, parity, gravidity and offspring sex. Crude and adjusted ORs statistics, as well as a $95 \%$ CI, were calculated. All statistical analyses were performed using SAS V.9.2 (SAS Statistical Institute). 
Table 2 Association between vomiting and PTB and its association stratified by pre-pregnancy BMI

\begin{tabular}{|c|c|c|c|c|c|c|}
\hline & Preterm, n (\%) & Full-term, n (\%) & Unadjusted OR (95\% Cl) & p Value & Adjusted OR $(95 \% \mathrm{Cl})^{*}$ & $p$ Value \\
\hline \multicolumn{7}{|c|}{ Vomiting } \\
\hline Yes & $4978(5.25)$ & $89879(94.75)$ & $1.08(1.04$ to 1.11$)$ & $<0.001$ & 1.05 (1.02 to 1.09$)$ & $0.0048^{\star}$ \\
\hline No & $10911(4.90)$ & 211695 (95.10) & 1.00 & & 1.00 & \\
\hline \multicolumn{7}{|c|}{ Underweight } \\
\hline \multicolumn{7}{|c|}{ Vomiting } \\
\hline Yes & $810(4.49)$ & $17216(95.56)$ & 1.08 (1.05 to 1.17$)$ & 0.0042 & 1.08 (1.04 to 1.17$)$ & $0.0082 \dagger$ \\
\hline No & $2308(4.12)$ & $53700(95.86)$ & 1.00 & & 1.00 & \\
\hline \multicolumn{7}{|c|}{ Normal } \\
\hline \multicolumn{7}{|c|}{ Vomiting } \\
\hline Yes & $3610(5.22)$ & 65485 (94.78) & 1.06 (1.04 to 1.11$)$ & 0.0048 & 1.06 (1.02 to 1.01$)$ & $0.0058 \dagger$ \\
\hline No & 7473 (4.94) & $143761(95.06)$ & 1.00 & & 1.00 & \\
\hline \multicolumn{7}{|c|}{ Overweight } \\
\hline \multicolumn{7}{|c|}{ Vomiting } \\
\hline Yes & $462(7.04)$ & $6099(92.96)$ & 1.02 (0.91 to 1.15$)$ & 0.7012 & 1.01 (0.90 to 1.14$)$ & $0.8547 \dagger$ \\
\hline No & $898(6.89)$ & $12127(93.11)$ & 1.00 & & 1.00 & \\
\hline \multicolumn{7}{|c|}{ Obese } \\
\hline \multicolumn{7}{|c|}{ Vomiting } \\
\hline Yes & $106(8.95)$ & $1079(91.05)$ & 0.93 (0.73 to 1.19$)$ & 0.5719 & 0.93 (0.73 to 1.19$)$ & $0.5508 \dagger$ \\
\hline No & $222(9.53)$ & 2107 (90.47) & 1.00 & & 1.00 & \\
\hline
\end{tabular}

*Adjusted for maternal age, education, parity, gravidity, pre-pregnancy BMI and offspring sex.

†Adjusted for maternal age, education, parity, gravidity and offspring sex.

\section{RESULTS}

In 317463 pregnancies included in our study (figure 1), gestational age ranged from 28 weeks to 43 weeks. Altogether, 94857 out of 317463 pregnant women (29.88\%) reported vomiting in the first trimester. Further, $5.00 \%$ (15 889) of the births were delivered preterm. Maternal demographics and characteristics are shown in table 1. Women who were older than 35 years, had a college education, were multipara, had more than three times gravidity or had a higher pre-pregnancy BMI were more likely to have a PTB. PTB was observed to be more common in female babies than in male offspring.

When vomiting women were compared with non-vomiting women, we found that vomiting in the first trimester produced a significant increase in risk for PTB, with $\mathrm{OR}=1.08$ and $95 \%$ CI 1.04 to 1.13 . The relationship still persisted $(\mathrm{OR}=1.05$, 95\% CI 1.02 to 1.09 , table 2) after adjustment for aforementioned confounders. In the additional stratified analyses, the results showed that vomiting in the first trimester was associated with PTB in underweight women $(\mathrm{OR}=1.08,95 \%$ CI 1.04 to 1.17 ) and in women with a normal pre-pregnancy BMI (OR=1.06, 95\% CI 1.02 to 1.11$)$, but not among overweight and obese groups. After adjusting for the same set of confounders, no changes occurred in the results (table 2).

Additionally, we assessed the associations of maternal age, education, parity, gravidity, pre-pregnancy BMI and offspring sex with risk of PTB. PTB was associated with all of the evaluated exposures (online supplementary table $\mathrm{S} 1$ ).

\section{DISCUSSION}

NVP while pregnant has far reaching effects on the mental and physical health of mothers and their offspring. Little research has accrued on the association between NVP and PTB in China, especially taking into account pre-pregnancy BMI. This study showed that nearly $30 \%$ of Chinese pregnant women had experienced vomiting in the first trimester. The rate of vomiting in Chinese women was somewhat different than in other studies. ${ }^{91125-27} \mathrm{~A}$ Norwegian cohort study reported that the rate of NVP was 33\% among 51675 women with 15 gestational weeks, ${ }^{9}$ which was higher than our findings. A previous meta-analysis estimated that the rate of NVP in the USA was $68.6 \%$ including 23 studies composed of 67602 women. ${ }^{11}$ Conflicting results can likely be explained by heterogeneity of populations, methods, definitions and confounders.

Our results showed that sociodemographic factors, such as age, education level, parity and gravidity, might influence PTB. Previous studies have indicated that women with advanced maternal age were associated with increased risk of PTB. ${ }^{28}{ }^{29}$ Araya et al reported that age $>35$ years, delivery of more than two fetuses and $<8$ years of education were risk factors for PTB. ${ }^{29}$ Women aged $>35$ years had longer exposure times to chronic pathologies and unhealthy lifestyles ${ }^{30}$ and higher prevalence of maternal obesity in older mothers, which were associated with risk factors for PTB. ${ }^{31}$ Several studies demonstrated that the lower the socioeconomic and education level, the 
higher the probability of developing infection, and that was clearly associated with PTB before 30 weeks of gestation. 3233

In this large cohort study conducted among Chinese women, we have found an association between vomiting in the first trimester and PTB before 37 weeks of gestation. When compared with non-vomiting pregnant women, women who experienced vomiting were at an increased risk of PTB. Our results were inconsistent with that of other studies. ${ }^{15} 183435$ For example, Czeizel and Puhó indicated that there was a significant association of vomiting with a decreased risk of PTB,${ }^{20}$ and Naumann $e t$ al found that vomiting was not associated with PTB. ${ }^{27}$ The differences found among these studies may be attributed to race, definition or classification of vomiting and the differential sample sizes of the populations mentioned above.

The possible causal link between vomiting and PTB remains elusive. The most common hypothesis proffered to explain the harmful effects of vomiting is based on the fact that vomiting leads to abnormal digestive function, which results in a lower nutrient intake of pregnant women. ${ }^{146}$ Vomiting may also affect maternal and fetal physiology through dehydration and the modulation of stress-related risk factors. ${ }^{37}{ }^{38}$ Moreover, vomiting may cause low maternal weight gain during pregnancy, which has been associated with PTB as reported by Canadian research. ${ }^{6}$

Additionally, our results reveal that vomiting was a risk factor for PTB in women with underweight and normal pre-pregnancy BMI, but not in overweight and obese women. Previous studies have estimated the risk factors for vomiting and PTB separately and found different effects of pre-pregnancy BMI on vomiting and PTB. ${ }^{39-41}$ No study has assessed the association between vomiting and PTB by pre-pregnancy BMI. The biological mechanisms underlying the associations with maternal pre-pregnancy BMIs remain unclear. Shaw et al indicated that the relationship between pre-pregnancy BMI and risk of PTB was complex. ${ }^{23}$ Although it was not statistically significant, a trend towards lower risk for PTB in obese women with vomiting was observed. We speculate that vomiting may have relatively few impacts on the nutrition of obese women due to their increased capacity for energy storage.

Strengths of this study are as follows. First, this is the first study exploring the relationship between vomiting in the first trimester and PTB in Chinese women while assessing the association between PTB and vomiting by pre-pregnancy BMI status. Second, the data were collected from the large population-based cohort and linked to the WMCHMIS, providing thorough and detailed access to pregnancy and birth outcomes. Third, with such a large cohort size, many significant associations tend to appear, and the merit of these in the clinical setting is notable. However, there are two limitations. One is the reliance on self-reported data on vomiting. Retrospective evaluation of vomiting symptoms has been reported as a possible source of bias. ${ }^{42}$ The other is that the prevalence of overweight and obesity was relatively low in our sample $(6.17 \%$ and $1.10 \%$, respectively), and the relationship between vomiting and PTB in women with a high pre-pregnancy BMI needs to be further assessed.

\section{CONCLUSION}

This study showed that vomiting presented an increased risk for PTB based on a large cohort study in China, although the effect was small. Vomiting was a risk for PTB in women who were underweight and normal weight based on their pre-pregnancy BMI. Although no significant association between vomiting and PTB in overweight and obese women was observed, clinicians should give all women suitable guidance for dealing with vomiting to ensure improved pregnancy outcomes. Finally, we recognise the need for greater clarity with respect to the association between vomiting and PTB and encourage researchers to build on our findings.

Acknowledgements We are extremely grateful to all the families who took part in this study, the staff of the Wuhan Health Bureau and all the hospitals and community health centres involved in this study.

Contributors $\mathrm{RH}, \mathrm{YC}$ and $\mathrm{BZ}$ designed the research. $\mathrm{YC}, \mathrm{YZ}$ and $\mathrm{ML}$ analysed the data. $\mathrm{RH}, \mathrm{YC}$ and $\mathrm{YL}$ drafted the manuscript. ZQ, MGV, SX, TZ and BZ revised the manuscript.

Competing interests None declared.

Patient consent Obtained.

Ethics approval The Institutional Review Board of Wuhan Children's Hospital (Wuhan Maternal and Child Healthcare Hospital) received and approved this study (approved on 13 October 2016).

Provenance and peer review Not commissioned; externally peer reviewed.

Data sharing statement № additional data are available.

Open Access This is an Open Access article distributed in accordance with the Creative Commons Attribution Non Commercial (CC BY-NC 4.0) license, which permits others to distribute, remix, adapt, build upon this work non-commercially, and license their derivative works on different terms, provided the original work is properly cited and the use is non-commercial. See: http://creativecommons.org/ licenses/by-nc/4.0/

(C) Article author(s) (or their employer(s) unless otherwise stated in the text of the article) 2017. All rights reserved. No commercial use is permitted unless otherwise expressly granted.

\section{REFERENCES}

1. Flaxman SM, Sherman PW. Morning sickness: a mechanism for protecting mother and embryo. Q Rev Biol 2000;75:113-48.

2. Koren G, Madjunkova S, Maltepe C. The protective effects of nausea and vomiting of pregnancy against adverse fetal outcome-a systematic review. Reprod Toxicol 2014;47:77-80.

3. Gadsby R, Barnie-Adshead AM, Jagger C. A prospective study of nausea and vomiting during pregnancy. $\mathrm{Br} J$ Gen Pract 1993;43:245-8.

4. Mazzotta P, Maltepe C, Navioz Y, et al. Attitudes, management and consequences of nausea and vomiting of pregnancy in the United States and Canada. Int J Gynaecol Obstet 2000;70:359-65.

5. Smith C, Crowther C, Beilby J, et al. The impact of nausea and vomiting on women: a burden of early pregnancy. Aust N Z J Obstet Gynaecol 2000;40:397-401.

6. Dodds L, Fell DB, Joseph KS, et al. Outcomes of pregnancies complicated by hyperemesis gravidarum. Obstet Gynecol 2006;107(2 Pt 1):285-92.

7. Einarson TR, Piwko C, Koren G. Quantifying the global rates of nausea and vomiting of pregnancy: a meta analysis. J Popul Ther Clin Pharmacol 2013;20:e171-83. 
8. Koch KL, Frissora CL. Nausea and vomiting during pregnancy. Gastroenterol Clin North Am 2003;32:201-34.

9. Chortatos $\mathrm{A}$, Haugen $\mathrm{M}$, Iversen $\mathrm{PO}$, et al. Pregnancy complications and birth outcomes among women experiencing nausea only or nausea and vomiting during pregnancy in the Norwegian Mother and Child Cohort Study. BMC Pregnancy Childbirth 2015;15:138.

10. Källén B, Lundberg G, Aberg A. Relationship between vitamin use, smoking, and nausea and vomiting of pregnancy. Acta Obstet Gynecol Scand 2003;82:916-20.

11. Einarson TR, Piwko C, Koren G. Prevalence of nausea and vomiting of pregnancy in the USA: a meta analysis. $J$ Popul Ther Clin Pharmacol 2013;20:e163-70.

12. Chin RK. Antenatal complications and perinatal outcome in patients with nausea and vomiting-complicated pregnancy. Eur J Obstet Gynecol Reprod Biol 1989;33:215-9.

13. Chan OK, Sahota DS, Leung TY, et al. Nausea and vomiting in health-related quality of life among Chinese pregnant women. Aust $N$ Z J Obstet Gynaecol 2010;50:512-8.

14. Birkeland E, Stokke G, Tangvik RJ, et al. Norwegian PUQE (Pregnancy-Unique Quantification of Emesis and nausea) identifies patients with hyperemesis gravidarum and poor nutritional intake: a prospective cohort validation study. PLoS One 2015;10:e0119962.

15. Roseboom TJ, Ravelli AC, van der Post JA, et al. Maternal characteristics largely explain poor pregnancy outcome after hyperemesis gravidarum. Eur J Obstet Gynecol Reprod Biol 2011;156:56-9.

16. Chan RL, Olshan AF, Savitz DA, et al. Severity and duration of nausea and vomiting symptoms in pregnancy and spontaneous abortion. Hum Reprod 2010;25:2907-12.

17. Paauw JD, Bierling S, Cook CR, et al. Hyperemesis gravidarum and fetal outcome. JPEN J Parenter Enteral Nutr 2005;29:93-6.

18. Temming L, Franco A, Istwan N, et al. Adverse pregnancy outcomes in women with nausea and vomiting of pregnancy. J Matern Fetal Neonatal Med 2014;27:84-8.

19. Zhang J, Cai WW. Severe vomiting during pregnancy: antenatal correlates and fetal outcomes. Epidemiology 1991;2:454-7.

20. Czeizel AE, Puhó E. Association between severe nausea and vomiting in pregnancy and lower rate of preterm births. Paediatr Perinat Epidemiol 2004;18:253-9.

21. Zhou A, Xiong C, Hu R, et al. Pre-pregnancy BMI, gestational weight gain, and the risk of hypertensive disorders of pregnancy: a cohort study in Wuhan, China. PLoS One 2015;10:e0136291.

22. Wang Y, Mi J, Shan XY, et al. Is China facing an obesity epidemic and the consequences? The trends in obesity and chronic disease in China. Int J Obes2007;31:177-88.

23. Shaw GM, Wise PH, Mayo J, et al. Maternal prepregnancy body mass index and risk of spontaneous preterm birth. Paediatr Perinat Epidemiol 2014;28:302-11.

24. Vikanes ÅV, Støer NC, Magnus P, et al. Hyperemesis gravidarum and pregnancy outcomes in the Norwegian Mother and Child Cohort-a cohort study. BMC Pregnancy Childbirth 2013;13:169.

25. Klebanoff MA, Koslowe PA, Kaslow R, et al. Epidemiology of vomiting in early pregnancy. Obstet Gynecol 1985;66:612-6.
26. Tierson FD, Olsen CL, Hook EB. Nausea and vomiting of pregnancy and association with pregnancy outcome. Am J Obstet Gynecol 1986;155:1017-22.

27. Naumann CR, Zelig C, Napolitano PG, et al. Nausea, vomiting, and heartburn in pregnancy: a prospective look at risk, treatment, and outcome. J Matern Fetal Neonatal Med 2012;25:1488-93.

28. Laopaiboon M, Lumbiganon P, Intarut N, et al. Advanced maternal age and pregnancy outcomes: a multicountry assessment. BJOG 2014;121:49-56.

29. Araya BM, Díaz M, Paredes D, et al. Association between preterm birth and its subtypes and maternal sociodemographic characteristics during the post-transitional phase in a developing country with a very high human development index. Public Health 2017;147:39-46.

30. Chen X, Scholl TO. Association of elevated free fatty acids during late pregnancy with preterm delivery. Obstet Gynecol 2008;112:297-303

31. Passini R, Cecatti JG, Lajos GJ, et al. Brazilian multicentre study on preterm birth (EMIP): prevalence and factors associated with spontaneous preterm birth. PLoS One 2014;9:e109069.

32. Ovalle A, Kakarieka E, Rencoret G, et al. [Risk factors for preterm deliveries in a public hospital]. Rev Med Chil 2012;140:19--29.

33. Goldenberg RL, Culhane JF, lams JD, et al. Epidemiology and causes of preterm birth. Lancet 2008;371:75-84.

34. Veenendaal MV, van Abeelen AF, Painter RC, et al. Consequences of hyperemesis gravidarum for offspring: a systematic review and metaanalysis. BJOG 2011;118:1302-13.

35. Schiff MA, Reed SD, Daling JR. The sex ratio of pregnancies complicated by hospitalisation for hyperemesis gravidarum. BJOG 2004:111:27-30.

36. Lee J, Lee J, Lim H. Morning sickness reduces dietary diversity, nutrient intakes, and infant outcome of pregnant women. Nutr Res 2004;24:531-40.

37. Järnfelt-Samsioe A, Eriksson B, Waldenström J, et al. Some new aspects on emesis gravidarum. Relations to clinical data, serum electrolytes, total protein and creatinine. Gynecol Obstet Invest 1985;19:174-86.

38. Haugen M, Vikanes A, Brantsaeter AL, et al. Diet before pregnancy and the risk of hyperemesis gravidarum. Br J Nutr 2011;106:596-602

39. Huxley RR. Nausea and vomiting in early pregnancy: its role in placental development. Obstet Gynecol 2000;95:779-82.

40. Girsen Al, Mayo JA, Carmichael SL, et al. Women's prepregnancy underweight as a risk factor for preterm birth: a retrospective study. BJOG 2016;123:2001-7.

41. Pan $\mathrm{Y}$, Zhang S, Wang Q, et al. Investigating the association between prepregnancy body mass index and adverse pregnancy outcomes: a large cohort study of 536098 Chinese pregnant women in rural China. BMJ Open 2016;6:e011227.

42. Koren G, Maltepe C, Navioz Y, et al. Recall bias of the symptoms of nausea and vomiting of pregnancy. Am J Obstet Gynecol 2004;190:485-8. 